\title{
3D N-BODY SIMULATIONS OF THE MILKY WAY
}

\author{
R. FUX, L. MARTINET AND D. PFENNIGER \\ Geneva Observatory \\ Ch. des Maillettes 51, CH-1290 Sauverny, Switzerland
}

\section{Introduction}

While the existence of a central bar in our Galaxy now seems to be well established, its parameters (such as position angle, extension, axis ratio, angular speed etc...) still remain controversial. The large amount of photometrical and stellar kinematical data becoming now available within $\sim 30^{\circ}$ of the Galactic Centre should provide potentially new constraints on these parameters. Unfortunately, a detailed barred model of the Milky Way, which would offer a powerful work frame to interpret such observations, does not exist yet. We therefore report here on a first attempt at constructing a 3D dynamically self-consistent barred model of the Galaxy. The idea is to follow the time evolution of a set of 400,000 particles initially distributed according to a plausible axisymmetric mass model of the Milky Way and in virial equilibrium, hoping that a bar will form spontaneously. Gas is not included so far, but will be introduced as a next step in this ongoing work. Some results presented at this meeting will be only quickly summarised here and explained in some more details elsewhere (Fux et al. 1995 and $A \mathscr{E} A$ paper in preparation).

\section{Initial conditions}

The initial axisymmetric mass distribution includes three components (see Table 1): a radially and vertically exponential disc, representing mainly the Galactic old disc (no division into subpopulations), a so-called nucleusspheroid (NS) with mass density scaling as $R^{-1.8}$ near the centre, as is observed in the near IR, and as $R^{-3.3}$ in the outer part, as is for example the case for the globular cluster system, and a dark halo (DH) to ensure a flat rotation curve up to at least $30 \mathrm{kpc}$. All parameters of this mass 
model have been adjusted to several observational constraints, assuming $R_{\circ}=8 \mathrm{kpc}$.

For each component, we have assumed an isotropic Maxwellian velocity distribution and solved numerically the hydrodynamical Jeans equations for the (isotropic) velocity dispersion $\sigma$ and the mean azimuthal velocity $\overline{u_{\phi}}$ (no streaming motion in the other directions). Before starting the main simulation, we let the DH relax for $3 \mathrm{Gyr}$ imposing axisymmetry, and the kinematics of the other components, kept fixed during that time, was in fact calculated with the final DH mass distribution. The resulting rotation curve and disc velocity moments are shown in Fig.2a.

TABLE 1. Initial mass model of the simulation. The scale length of the disc is taken from Robin et al. (1992) and Fux \& Martinet (1994). The oblate NS and DH have a common axis ratio $e=0.5$

\begin{tabular}{|c|c|c|c|}
\hline Component & \# particles & Space density & Parameters \\
\hline Disc & 150,000 & $\propto \exp \left(-R / h_{R}-z / h_{z}\right)$ & $\begin{array}{l}h_{R}=2.5 \mathrm{kpc} \\
h_{z}=250 \mathrm{pc} \\
M=4.6 \cdot 10^{10} \mathrm{M}_{\odot}\end{array}$ \\
\hline NS & 50,000 & $\begin{array}{l}\propto \frac{m^{p}}{1+m^{p-q}} \\
\quad m^{2}=\left(R^{2}+e^{2} z^{2}\right) / a^{2}\end{array}$ & $\begin{array}{l}p=-1.8, \quad q=-3.3 \\
a=1 \mathrm{kpc} \\
(M / L)_{K}=0.75 \text { (solar units) }\end{array}$ \\
\hline $\mathrm{DH}$ & 200,000 & $\begin{array}{l}\propto \exp (-\mu) \\
\qquad \mu^{2}=\left(R^{2}+e^{2} z^{2}\right) / b^{2}\end{array}$ & $\begin{array}{l}b=9.1 \mathrm{kpc} \\
M=3.2 \cdot 10^{11} \mathrm{M}_{\odot}\end{array}$ \\
\hline
\end{tabular}

\section{Time evolution}

The time integration is achieved using the polar-cylindrical PM code of Pfenniger \& Friedli (1993) with a central resolution of $38 \mathrm{pc}$ and a rather short time step of $0.1 \mathrm{Myr}$ because of the strong density peak of the NS component in the centre. We also impose two symmetries, namely reflexions about the $z$ axis and about the Galactic plane. The total integration time is $5 \mathrm{Gyr}$ and thus requires 50,000 time-steps.

The simulation, illustrated in Fig.1, leads to the formation of a bar after about $1 \mathrm{Gyr}$ and then strong spiral arms appear and persist until about 2 Gyr. The extension of the bar is $\sim 5 \mathrm{kpc}$ and its axis ratio in the plane $\sim 0.5$. As one could expect, the spiral arms do heat the disc very efficiently: its radial velocity dispersion almost doubles from 1 to 2 Gyr, while its scale height outside the bar region increases by a factor 1.3 . However, the innermost disc $(R<1 \mathrm{kpc})$ does not follow this thickening and surprisingly 

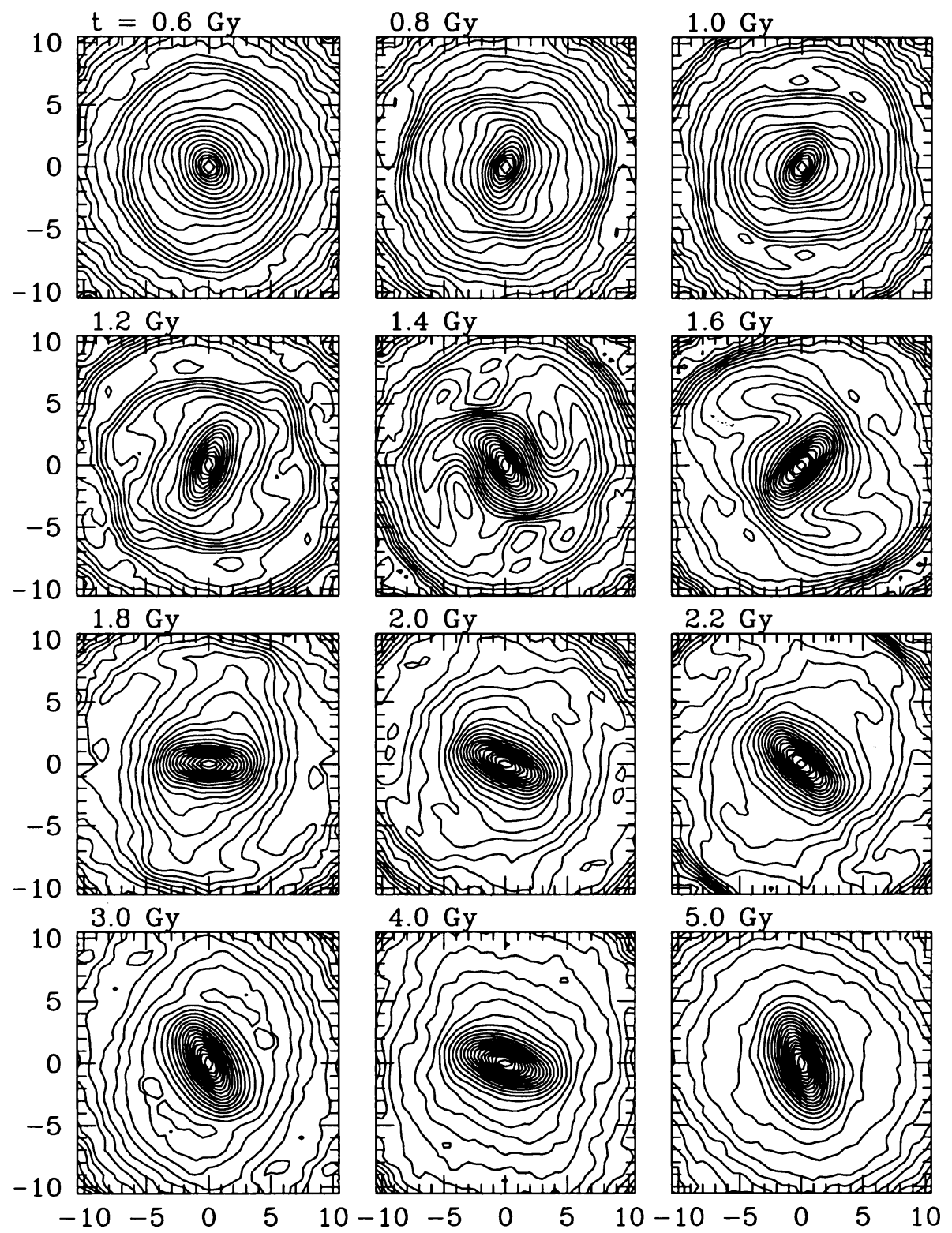

Figure 1. Face-on isodensity curves of the visible components at several times. The length unit is one kpc

becomes the thinnest part of the whole disc.

The velocity dispersion of the disc is closest to the observed one for the Galactic old disc stars at about $1.2 \mathrm{Gyr}$ (see Fig.2b) and then the disc becomes substantially too hot (we expect however that a gas component, 
not included in the simulation yet, will reduce this heating). In Fig.2 we also notice that, although we have started with a rather unrealistic isotropic velocity dispersion, the kinematics of the disc evolves spontaneously to anisotropy, with the components of the velocity dispersion just ordered in the same way as observed in the solar neighbourhood, i.e. $\sigma_{R R}>\sigma_{\phi \phi}>\sigma_{z z}$.

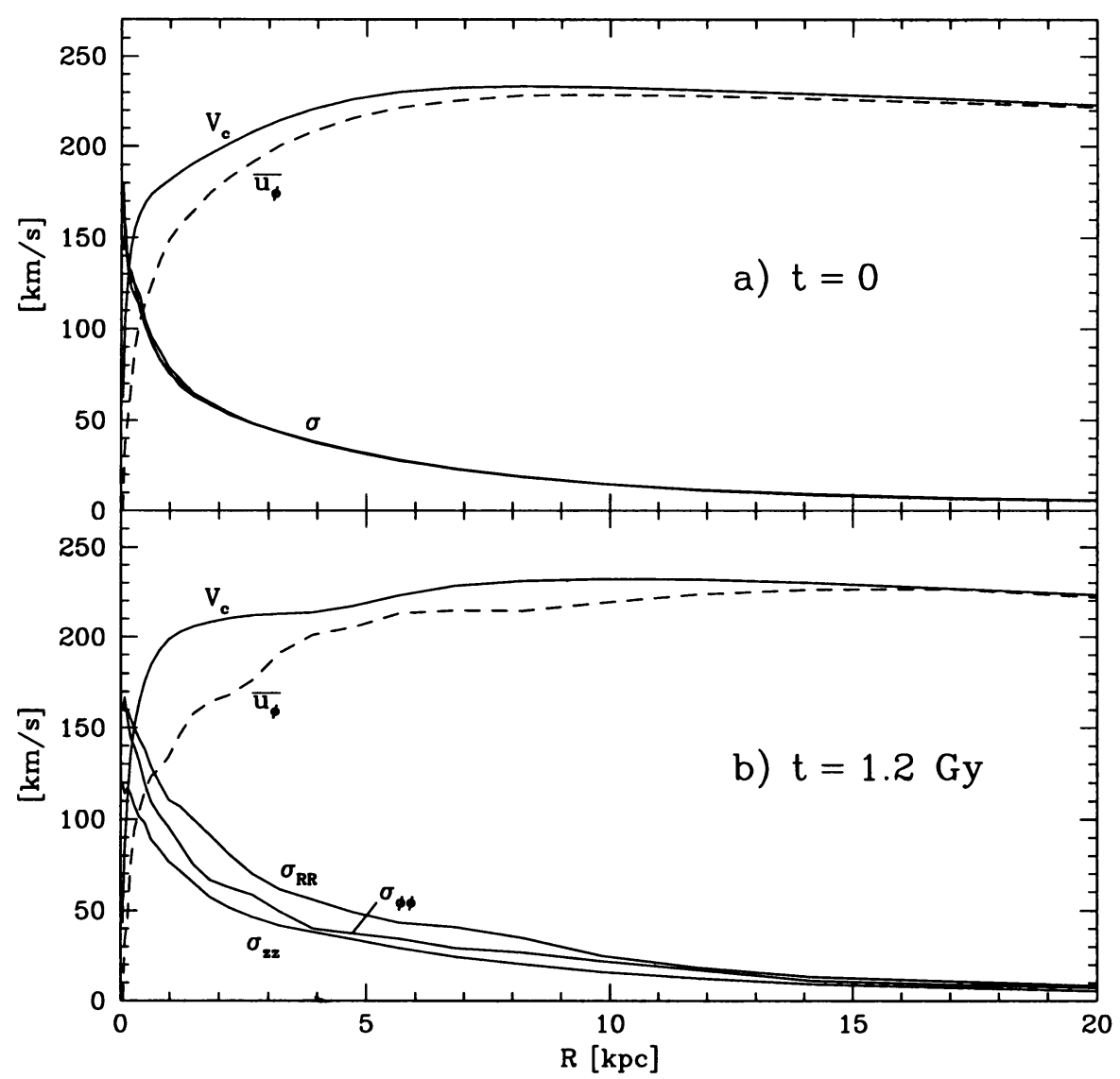

Figure 2. Rotation curve $\left(V_{c}\right)$, and mean azimuthal motion $\left(\overline{u_{\phi}}\right)$ and velocity dispersion $\left(\sigma_{i i}\right)$ of the disc

\section{Kinematics along Baade's Window}

The central radial velocity dispersion of the disc in Fig.2b is not directly comparable to existing observational data in Baade's Window, for example 
those given by Lewis and Freeman (1989) for K giants, because the line of sight through this field passes more than 500 pc away from the Galactic Centre. The radial velocity dispersion computed so as to mimic observations in Baade's Window is shown in Fig.3. The result depends within $30 \%$ on the position angle of the fictive observer with respect to the bar, the maximum velocity dispersion occurring when the bar is seen end-on. All viewing angles lead to lower velocity dispersions than at $R=0$.

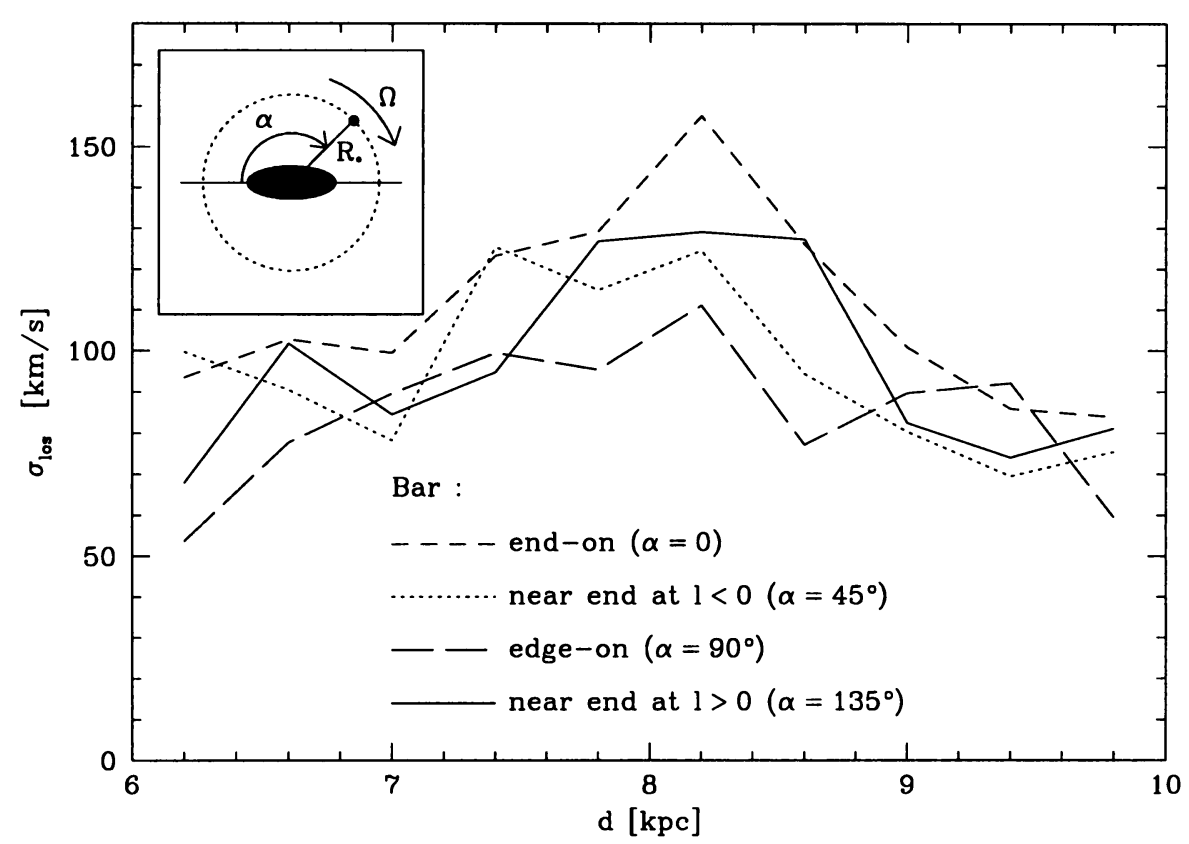

Figure 3. Line of sight velocity dispersion at $t=1.2 \mathrm{Gyr}$ when looking towards Baade's Window $\left(l=0.9^{\circ}, b=-3.9^{\circ}\right)$ from points on the solar circle with various position angles relative to the bar. $d$ is the distance from the observing point $(d \approx 8 \mathrm{kpc}$ close to the Galactic Centre) and $\alpha$ is the angle defined in the small embedded box

\section{Isophotes from the Sun's position}

During the simulation, the bar pumps disc stars into a peanut bar/bulge over a time scale of 1 Gyr. Fig.4 gives the "isophotes" as they would appear to our fictive observer moving along the solar circle a few Gyr after the formation of the bar. The peanut shape is clearly visible in the edge-on case and the intermediate case with the near end of the bar in the first Galactic quadrant qualitatively resembles COBE's image. 

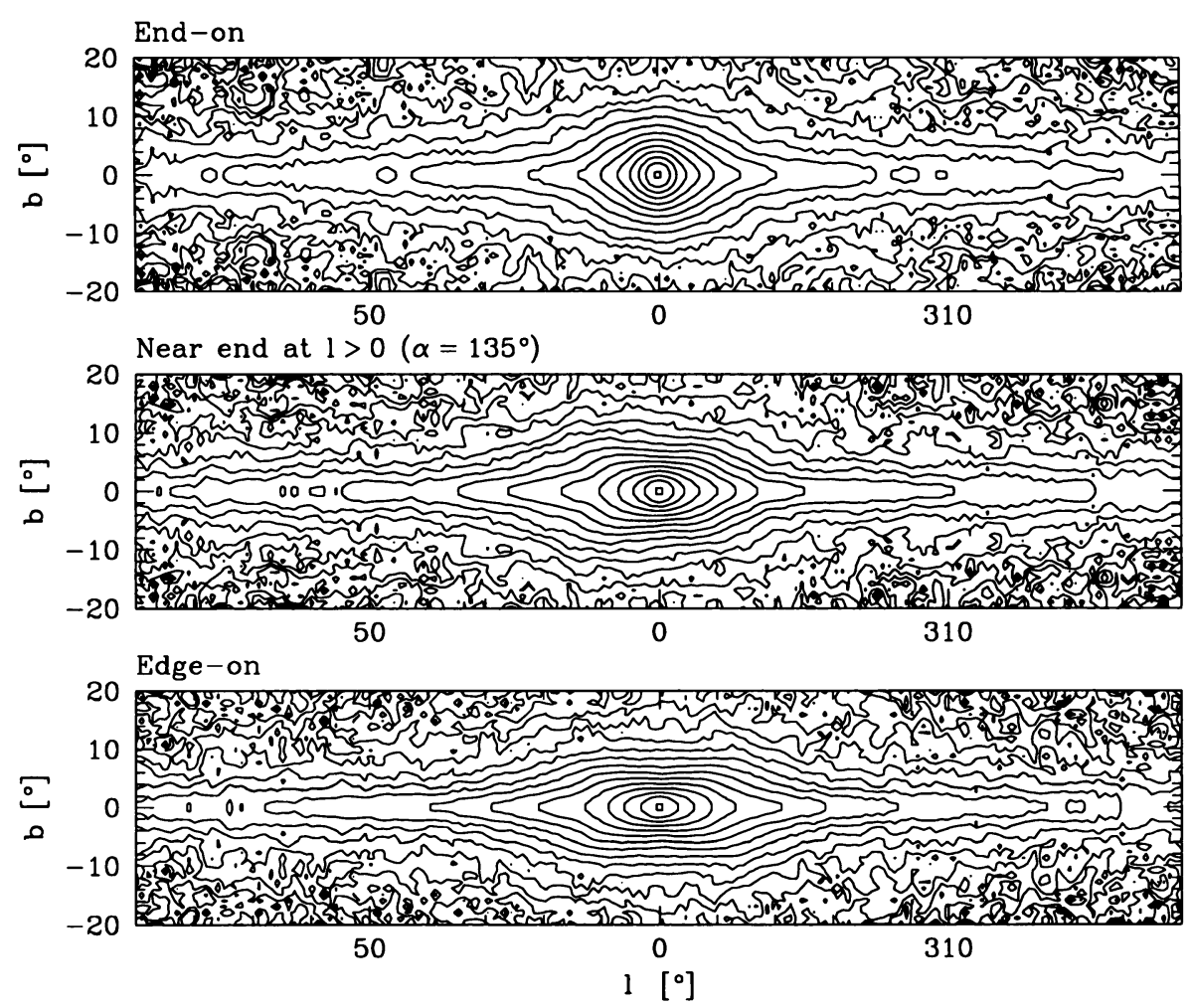

Figure 4. Surface brightness contours of the simulation at $t=5 \mathrm{Gyr}$ from various view points located on the solar circle. The contours are obtained from the visible components, assuming that the apparent luminosity of each particle is proportional to its mass divided by the square of its distance

\section{Other results}

- The corotation radius is close to $5 \mathrm{kpc}$ and the bar angular speed, of $45 \mathrm{~km} / \mathrm{s} / \mathrm{kpc}$ at $t=2 \mathrm{Gyr}$, decreases by $\sim 5 \mathrm{~km} / \mathrm{s} / \mathrm{kpc}$ every Gyr.

- From the velocity field analysis all around the solar circle in our simulation and the commonly accepted position angle of the Sun relative to the bar in the Milky Way (i.e. $\alpha \sim 135^{\circ}$ ), one could expect an outward mean radial motion of the disc stars in the Solar Neighbourhood.

\section{References}

Fux, R. and Martinet, L. 1994, $A \& A, 287$, L21

Fux, R., Martinet, L. and Pfenniger, D. 1995, in Proceedings: The Formation of the Milky Way, Granada, september 1994, Cambridge University Press

Lewis, J.R. and Freeman, K.C. 1989, $A J$, 97, 139

Pfenniger, D. and Friedli, D. 1993, $A \& A, 270,561$

Robin, A.C., Crézé, M. and Mohan, V. 1992, $A \mathscr{E} A, 265,32$ 


\section{DISCUSSION}

J. Palous: May we conclude anything about the bar from the observed vertex deviation of A stars in the solar vicinity?

Fux: The disc in our simulation does not separate into subpopulations like the young A stars. We may therefore only investigate the possible connection between the bar and the local kinematical properties of the dominant disc population, i.e. the old disc stars. The results will be published in a future paper. 